\title{
ScIDice
}

\author{
International Journal of Dentistry and Oral Science (IJDOS) \\ ISSN: $2377-8075$
}

\section{Effectiveness of Splint Therapy in the Management of Temporomandibular Joint Disorders}

Research Article

Ananya R ${ }^{1}$, M.P. Santhosh Kumar ${ }^{*}$, Nashwah Hinaz ${ }^{1}$

${ }^{1}$ Department of Oral and Maxillofacial Surgery, Saveetha Dental College and Hospitals, Saveetha Institute Of Medical and Technical Science, Saveetha University, Chennai, India.

${ }^{2}$ Reader, Department of Oral and Maxillofacial Surgery, Saveetha Dental College, Saveetha Institute Of Medical and Technical Science, Saveetha University, Chennai, India.

\section{Abstract}

Objectives: Temporomandibular joint disorders (TMDs) are a set of clinical diseases that involve the temporomandibular joint (TMJ), myofascial muscles, and other related tissues. There is currently no single standard for the classification of TMD, although the most widely used criteria are the research diagnostic criteria for temporomandibular disorders (RDC/TMD).TMJ pain and clicking, myofascial or oral masticatory muscle pain, and irregular jaw movement are the most common signs and symptoms of TMD. It is a major public health issue since it is a leading cause of persistent oral and facial pain that interferes with daily activities. Splint therapy is one of the conservative modalities for the management of temporomandibular joint disorders. The aim of this study was to assess the effectiveness of splint therapy in patients with temporomandibular joint disorders in our regional population.

Methods: In this retrospective study, a total of 192 patients who had temporomandibular disorders and treated with splint therapy were included. The following parameters were evaluated based on the dental records; age and gender of the patients, types of TMD, clinical manifestations of TMD, treatment outcomes, effectiveness of splint therapy, and types of splint therapy. Excel tabulation and SPSS version 23 was used for data analysis and results obtained.

Results: In our study, a total of 192 patients with TMD undergoing splint therapy were assessed with the age range of 10-60 years and mean age of $21-40$ years. The age group of $21-30$ years had higher rate of splint therapy $(37.82 \%)$ followed by $31-40$ years age group with $32.12 \%$. Male patients were predominantly on splint therapy (54.9\%) than the females $(45.08 \%)$ Splint therapy was predominantly provided for disc-condyle disorder patients followed by MPDS condition and least for the patients with degenerative disorders. Soft splint was predominantly $(89.2 \%)$ chosen for patients with TMD with only $10.8 \%$ patients were provided with hard splint as it was required. Splint therapy was effective for majority of the patients $(80 \%)$ with TMD. The association between age and gender of patients with the effectiveness of splint therapy for treating temporomandibular joint disorders was statistically significant. The association between type of splint therapy and effectiveness of splint therapy for treating temporomandibular joint disorders was statistically significant. Soft splints were more effective $(70.98 \%)$ than the hard splints $(8.29 \%)$. The association between type of temporomandibular joint disorder and effectiveness of splint therapy for treating temporomandibular joint disorders was statistically significant. Splint therapy was effective in $45 \%$ of patients with disc-condyle disorder and in $26.7 \%$ of patients with MPDS.It was not very useful in patient with degenerative disorders as it was effective in only $7.33 \%$ of patients.

Conclusion: In our study, an increased number of people in the younger age, especially males received the splint therapy for the management of temporomandibular joint disorders. Soft splints were chosen for the vast majority of the people which were very effective in treating temporomandibular joint disorders. Splint therapy was very effective in treating younger age people than the elderly. Splint therapy was effective for both females and males with males exhibiting a higher response rate. Splint therapy was very effective in treating patients with disc-condyle disorder followed by MPDS patients. In our study, majority of the patients with TMJ pain responded well to splint therapy, but a small a percentage of patients developed refractory or persistent TMD.

Keywords: Bruxism; Crepitus; Joint Pain; Temporomandibular Joint Disorders; Occlusal Splints; Trismus; MPDS; Clicking; Internal Derangement.

\section{*Corresponding Author:}

Dr. M P Santhosh Kumar M.D.S,

Reader, Department of Oral and Maxillofacial Surgery, Saveetha Dental College, Saveetha Institute Of Medical and Technical Science, Saveetha University, Chennai, India. Tel: 9994892022

E-mail: santhoshsurgeon@gmail.com

Received: May 28, 2021

Accepted: June 17, 202

Published: June 24, 2021

Citation: Ananya R, M.P. Santhosh Kumar, Nashwah Hinaz. Effectiveness of Splint Therapy in the Management of Temporomandibular Joint Disorders. Int J Dentistry Oral Sci. 2021;8(6):2792-2798. doi: http://dx.doi.org/10.19070/2377-8075-21000546

Copyright: M P Santhosh Kumar 2021. This is an open-access article distributed under the terms of the Creative Commons Attribution License, which permits unrestricted use, distribution and reproduction in any medium, provided the original author and source are credited. 


\section{Introduction}

Temporomandibular joint disorders (TMD) and its relationship to dentistry has been an important topic in recent years. Costen was the first to describe the temporomandibular joint (TMJ) condition in 1934. TMD was defined by the American Dental Association President's Conference on Temporomandibular Disorders (American Dental Association, 1983) as a set of orofacial disorders characterized by discomfort in the preauricular area, TMJ, or masticatory muscles, and a limitation in chewing ability [1].

TMD is a group of diseases characterized by discomfort in the TMJ or surrounding tissues, mandibular functional restrictions, or clicking in the TMJ during motion [2]. Temporomandibular dysfunction syndrome, pain dysfunction syndrome, facial arthromyalgia, TMJ dysfunction syndrome, and myofascial pain dysfunction are some of the terms used to describe conditions that cause pain and malfunction in the TMJ [3]. TMD is a frequent illness that affects people between the ages of 20 and 40 [4]. TMDs are a set of clinical diseases that involve the temporomandibular joint (TMJ), myofascial muscles, and other related tissues [5]. There is currently no single standard for the classification of TMD, although the most widely used criteria are the research diagnostic criteria for temporomandibular disorders (RDC/TMD).TMJ pain and clicking, myofascial or oral masticatory muscle pain, and irregular jaw movement are the most common indications and symptoms [6]. TMD is a major public health issue since it is a leading cause of persistent oral and facial pain that interferes with daily activities $[7,8]$. Other symptoms affecting the head and neck region, such as headache and ear-related problems, are frequently connected with these illnesses.Approximately, 33\% of the population has at least one TMD symptom, and $3.7 \%$ to $7 \%$ of the population has TMD that is severe enough to demand treatment [9]. TMJ issues have an unknown origin, however it is likely complex.Abnormal occlusion, parafunctional habits (e.g., bruxism [teeth grinding], teeth clenching, lip biting), stress, anxiety, or anomalies of the intra-articular disc can induce capsule inflammation or destruction, as well as muscle discomfort or spasm [9, 10]. People with and without TMJ symptoms both appear to have abnormal oral occlusion. TMJ microtrauma is hypothesized to be caused by non-functional habits [9-11].

Notably, whereas 60-70 percent of the general population has indicators of TMD, only around one-fourth of those who have indications are aware of any symptoms. Occlusal anomalies, psychological stress, orthodontic treatment, microtrauma, poor health and nutrition, joint laxity, and exogenous estrogen are some of the contributing factors to TMD [12]. Occlusal therapy, psychotherapy, physical therapy, medication, manual therapy, and surgery are the most common treatments for TMD. In practice, an occlusal splint is a removable appliance made of resin that is designed to cover all of the occlusal and incisal surfaces of the upper and lower jaw's teeth.Because of its ease of use, low cost, and broad indications, occlusal splint therapy is the most often utilized clinical method [6,12]. A prior meta-analysis looked at the efficacy of splint therapy for TMD, but why it works is still unknown.To learn more about the clinical success of splint therapy in the treatment of TMD in adults a meta-analysis was conducted which compared the clinical effects reported in all relevant randomized controlled trials to determine the functional features of splint therapy (RCTs) [13]. Splints can be used to treat or control
TMD in a variety of ways which includesthe stabilization splint, soft splint, flat splint, and pivot splint. Although the mechanisms of splint action are unknown, it was discovered that TMD patients treated with an occlusal splint had higher plasma levels of calcitonin gene related peptide (CGRP) $[4,13]$.

Medication, habit change, psychotherapy, physical therapy, splint therapy, and manipulation are examples of conservative treatments. Patients utilize medications like ketorolac and paracetamol for transient relief because of their effectiveness. Arthrocentesis, arthroscopy, arthroplasty, discectomy, and temporomandibular joint (TMJ) reconstruction are some of the surgical options. Patient with TMJ dysfunction who have orthognathic surgery are likely to have markedly better signs and symptoms after surgery [10]. Orthognathic surgery involves movement of the jaws in all three planes. It has been found that temporomandibular joint diseases can emerge as a result of maxillofacial trauma or road traffic incidents that directly or indirectly damage the TMJ. Oral splints (OS), also known as occlusal splints, are removable occlusal devices that are used to diagnose TMDs and treat the dysfunction of the mandible-maxilla relationship [10, 14, 15]. The initial choice for TMJ treatment is usually recognized to be a conservative, reversible approach. Oral splints are widely used for internal derangement and as one of the conservative therapies. Wearing anocclusal splint is thought to generate changes in mechanical sensitive input emerging from periodontal tissue and spindle afferents in the jaw closing muscle, as well as a decrease in intra-articular pressure in the TMJ, according to the mechanisms of occlusal splint therapy [16]. The occlusal splint is frequently effective, but the time it takes to achieve a pain-free normal range of motion is insufficient. Although dental splints are sometimes used to stabilize the occlusion or prevent dentition wear, one of the most prevalent uses in the treatment of TMDs is to protect the TMJ discs from dysfunctional stresses that can cause perforations or displacements [17].

Previous research has emphasized the relevance of conservative splint therapy in the treatment of TMD discomfort. While the investigation is being carried out, however, a number of difficulties are reported. Conservative splint therapy, according to Lee, HyeSung, et al, is extremely successful, however it does not always result in a good outcome [18]. In such circumstances, a surgical procedure should be explored. Some studies illustrate the necessity and need of splint therapy however, it takes a longer period of action to achieve a pain-free condition, and obtain patient compliance. Splint therapy is only effective in minor cases and cannot be used to treat complex TMD symptoms [19]. These appliances are frequently used in conjunction with other treatments like physiotherapy or medicines.

The goal of this study was to determine the effectiveness of splint therapy in patients with temporomandibular joint disorders and its association based on age and gender of the patients. The effectiveness of splint therapy in patients suffering from various temporomandibular joint disorders is assessed based on reduction in pain, discomfort and parafunctional habits, increased maximal mouth opening and improved quality of life.

\section{Materials And Methods}

Study design and Study setting 
This retrospective study was conducted in Saveetha dental college and hospital, Saveetha university, Chennai, to assess the effectiveness of splint therapy in the management of temporomandibular joint disorders among dental patients reporting from June 2019 to December 2020. The study was initiated after approval from the institutional review board andit was covered by the following ethical approval number; SDC/SIHEC/2020/DIASDATA/0619-0320.

\section{Study population and sampling}

Inclusion criteria for the study were adult dental patients with TMD. Exclusion criteria included immunocompromised patients, dental anomalies, systemic diseases with cognitive problems and speech problems, missing or incomplete data. After assessment in the university patient data registry, consecutive case records of 192 patients who were diagnosed with TMD and underwent splint therapy were included in the study. Cross verification of data for errors was done with the help of an external examiner.

\section{Data collection and tabulation}

Data regarding patients having TMD were retrieved after analysing 86000 case sheets. The following parameters were evaluated based on the dental records; age, gender, types of TMD, types of splint therapy for TMD. Chief complaints, medical and dental history,all the clinical manifestations of TMDs, treatment strategies andoutcomes of the patients with the splint therapy [effectiveness or ineffectiveness] were examined from the data, collected and recorded. Patients diagnosed with TMD were further classified into disc-condyle disorder [TMJ internal derangement,
TMJ dislocation/subluxation], degenerative disorder and myofascial pain and dysfunction syndrome (MPDS). The clinical presentations of TMD were considered into several variables and the patients were classified according to the severity of the TMD for diagnosis, treatment and prognosis purposes. Data was entered in excel and was imported to SPSS. The variables were defined.

\section{Statistical Analysis}

The collected data was validated, tabulated and analysed with Statistical Package for Social Sciences for Windows, version 23.0 (SPSS Inc., Chicago, IL, USA) and results were obtained. Descriptive analysis was used to describe the number of patients treated with splint therapy based on age, gender, types of TMD, types of splint therapy and effectiveness of the splint therapy among the study population. Categorical variables were expressed in frequency and percentage; and continuous variables in mean and standard deviation. Chi-square test was used to test associations between categorical variables (age, gender, types of TMD, types of splint therapy, and effectiveness of splint therapy for treating temporomandibular joint disorders). $\mathrm{P}$ value $<0.05$ was considered statistically significant.

\section{Results}

In our study, a total of 192 patients with TMD undergoing splint therapy were assessed with the age range of 10-60 years and mean age of 21-40 years. The age group of 21-30 years had higher rate of splint therapy $(37.82 \%)$ followed by $31-40$ years age group with $32.12 \%$ [Figure 1]. Male patients were predominantly on splint therapy $(54.9 \%)$ than the females $(45.08 \%)$ [Figure 2]. Splint therapy was predominantly provided for disc-condyle disorder

Figure 1. Bar graph showing distribution of age of the patients undergoing splint therapy for temporomandibular joint disorders. The age group of 21-30 years shows more incidence of splint therapy (37.82\%) followed by 31-40 years age group with $32.12 \%$. $\mathrm{X}$ axis represents the age group and $\mathrm{Y}$ axis represents the number of patients undergoing splint therapy.

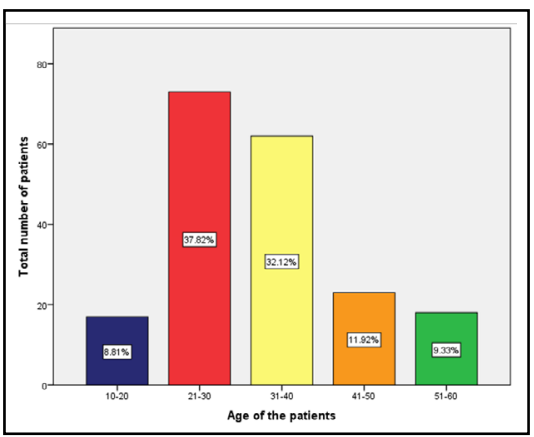

Figure 2. Bar graph showing distribution of gender of the patients undergoing splint therapy for temporomandibular joint disorders. Blue denotes male participants with $54.9 \%$ and red denotes female participants with $45.08 \%$. X axis represents the gender of patients and $\mathrm{Y}$ axis represents the number of patients undergoing splint therapy. Male patients were predominantly on splint therapy $(54.9 \%)$ than the females $(45.08 \%)$.

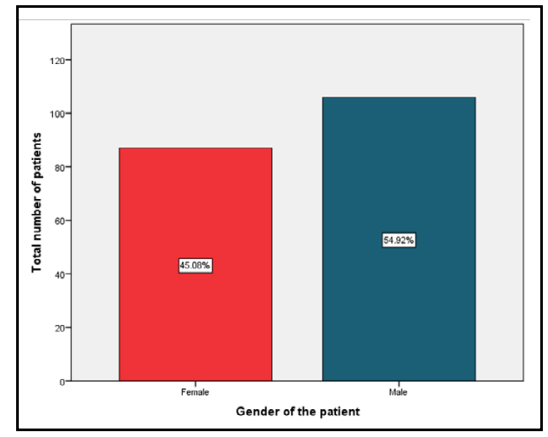


patients followed by MPDS condition and least for the patients with degenerative disorders [Figure 3]. Soft splint was predominantly $(89.2 \%)$ chosen for patients with TMD with only $10.8 \%$ patients were provided with hard splint as it was required [Figure 4]. Splint therapy was effective for majority of the patients $(80 \%)$ with TMD [Figure 5].

The association between age and gender of patients with the effectiveness of splint therapy for treating temporomandibular joint disorders was statistically significant. Effectiveness of splint therapy was higher in age group $21-30$ years (34.72\%), and it was (slightly) ineffective in $8.81 \%$ of patients in the age group of 31 40 years [Figure 6]. Splint therapy was effective for both females and males with $35.75 \%$ and $43.52 \%$ respectively with a higher rate for males [Figure 7]. The association between type of splint therapy and effectiveness of splint therapy for treating temporomandibular joint disorders was statistically significant. Soft splints were more effective $(70.98 \%)$ than the hard splints $(8.29 \%)$ [Figure 8]. The association between type of temporomandibular joint disorder and effectiveness of splint therapy for treatingtemporomandibular joint disorders was statistically significant. Splint therapy was effective in $45 \%$ of patients with disc-condyle disorder and in $26.7 \%$ of patients with MPDS. It was not very useful in patient with degenerative disorders as it was effective in only $7.33 \%$ of patients [Figure 9].

Figure 3. Bar graph showing distribution of splint therapy based on the types of temporomandibular joint disorders. Purple denotes 20 patients with degenerative disorder, orange denotes 107 patients with disc-condyle disorder, and blue denotes 64 patients with MPDS. $X$ axis represents the types of TMD and $Y$ axis represents the number of patients undergoing splint therapy. Splint therapy was predominantly provided for disc-condyle disorderpatients followed by MPDS condition and least for the patients with degenerative disorders.

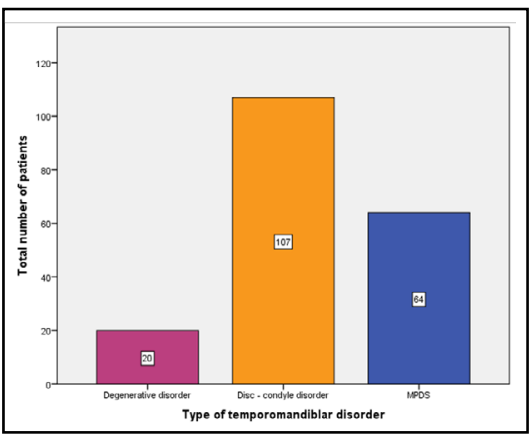

Figure 4. Bar chart showing distribution of types of splint therapy among the patients with temporomandibular joint disorder. Blue denotes hard splint therapy (anterior repositioning splint) with $\mathbf{1 0 . 8 \%}$ and red denotes soft splint therapy for treating TMD with $89.2 \%$. $\mathrm{X}$ axis represents the type of splints therapy in patients and $\mathrm{Y}$ axis represents the number of patients undergoing splint therapy. Soft splint was predominantly $(89.2 \%)$ chosen for patients with TMD with only $10.8 \%$ patients were provided with hard splint as it was required.

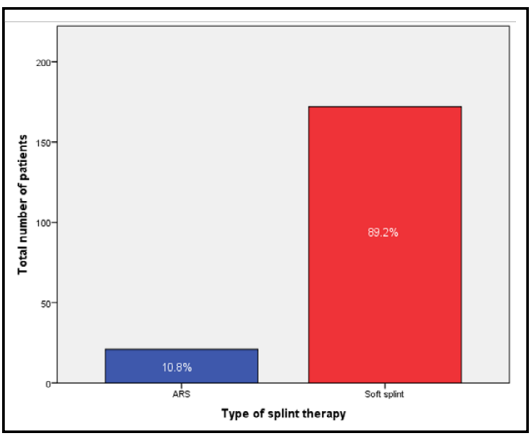

Figure 5. Bar chart showing distribution of effectiveness of splint therapy among the patients with temporomandibular joint disorder. Purple denotes effectiveness of splint therapy for TMD with $79.2 \%$ and yellow denotes ineffectiveness of splints therapy for TMD with $20.8 \%$. $\mathrm{X}$ axis represents the effectiveness of splints therapy in patients and $\mathrm{Y}$ axis represents the number of patients undergoing splint therapy. Splint therapy was effective for majority of the patients (80\%) with TMD.

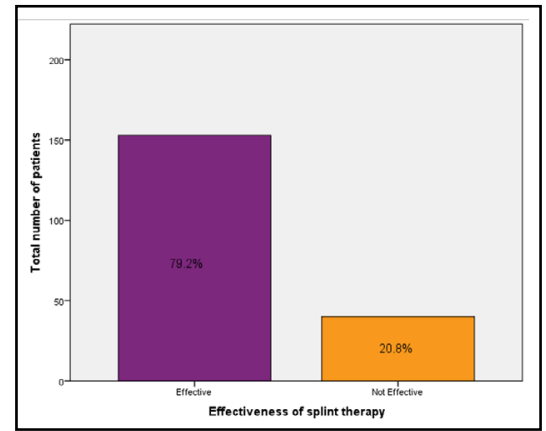


Figure 6. Bar graph depicting the association between age of patients and effectiveness of splint therapy for treating temporomandibular joint disorders. $\mathrm{X}$ axis represents the age of the patients and $\mathrm{Y}$ axis represents the effectiveness of splint therapy in patients with TMD. Effectiveness of splint therapy was higher in age group 21-30 years (34.72\%), and it was (slightly) ineffective in $8.81 \%$ of patients in the age group of $31-40$ years. (Pearson Chi square was done with $p=0.001$ $(<0.05)$, hence statistically significant). Thus, the association between age of patients and effectiveness of splint therapy was statistically significant.

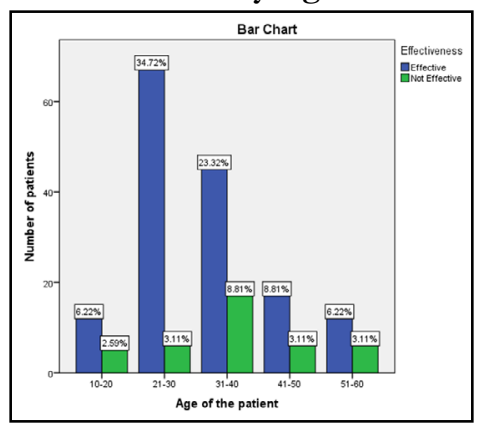

Figure 7. Bar graph showing association between gender and effectiveness of splint therapy for treating temporomandibular joint disorders. $\mathrm{X}$ axis represents the gender of the patients and $\mathrm{Y}$ axis represents the effectiveness of splint therapy in patients with TMD. Blue bars represent the effectiveness of splint therapy which is higher for both females and males with $35.75 \%$ and $43.52 \%$ respectively, and green bars represent non-effectiveness of splint therapy. Splint therapy was effective in both genders. Pearson Chi square was done with $p=0.038(<0.05)$, hence statistically significant. Thus, the association between gender of patients and effectiveness of splint therapy was statistically significant.

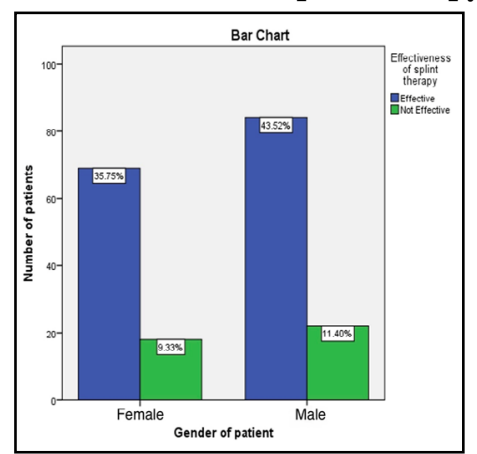

Figure 8. Bar graph showing association between type of splint therapy and effectiveness of splint therapy for treating temporomandibular joint disorders. $\mathrm{X}$ axis represents the type of splint therapy and $\mathrm{Y}$ axis represents the effectiveness of splint therapy in patients with TMD. Blue bars represent the effectiveness of splint therapy which is higher for soft splints with $70.98 \%$ and green bars represent non-effectiveness of splint therapy. Pearson Chi square was done with $p=0.025(<0.05)$, hence statistically significant. Thus, association between type of splint therapy and effectiveness of splint therapy was statistically significant. Soft splints were more effective $\mathbf{( 7 0 . 9 8 \% )}$ than the hard splints $(8.29 \%)$.

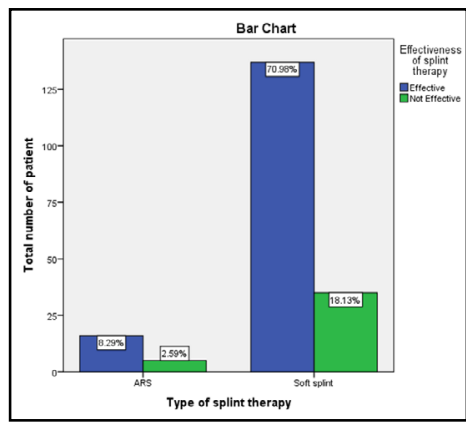

\section{Discussion}

TMJ disorders are now evolving commonly among the younger population. Disc-condyle disorders occurs more commonly than the other types of TMD.According to our study, the mean age of participants undergoing splint therapy was between 21-40 years. Similarlyin a study by Wassell et al, patients reporting temporomandibular joint disease were aged 18 and above, with a mean age of 30.2 years [20]. The average age of individuals reporting with TMD and for conservative management was 22.59 years in another study by Lee HS et al [18]. Thus, most of the studies show that the age range most affected by TMD is from 18 to 65 years of age. In a study on TMD, 45 of the 76 patients were males and 31 were females [3]. Similarlymale patients were predominantly on splint therapy $(54.9 \%)$ than the females $(45.08 \%)$ in our study. Lee HS., et al. in their study found that out of 43 cases, thirty were men and forty were women, contradicting our findings [18]. This difference in male to female ratio among the studies could be due to the variations in the small sample size and demographics.

Because most TMD symptoms remit quickly, usually within 2-4 weeks, conservative treatment is deemed more appropriate than 
Figure 9. Bar graph showing association between type of temporomandibular joint disorder and effectiveness of splint therapy for treating temporomandibular joint disorders. $\mathrm{X}$ axis represents the type of TMD and $\mathrm{Y}$ axis represents the effectiveness of splint therapy in patients with TMD. Blue bars represent the effectiveness of splint therapy which is higher in disc-condylar disorder with $45.03 \%$ and green bars represent non-effectiveness of splint therapy. Pearson Chi square was done with $\mathrm{p}=0.041(<0.05)$, hence statistically significant. Thus, theassociation between type of temporomandibular joint disorder and effectiveness of splint therapy was statistically significant.Splint therapy was effective in $45 \%$ of patients with disc-condyle disorder and in $26.7 \%$ of patients with MPDS. It was not very useful in patient with degenerative disorders as it was effective in only $7.33 \%$ of patients.

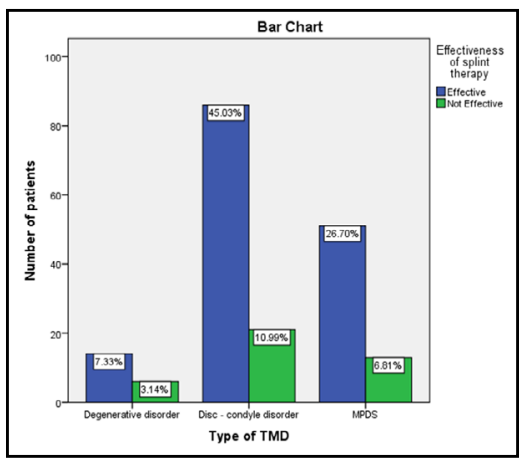

surgery for these conditions [21]. Soft splints have certain advantages as a conservative treatment for TMDs, such as their relative simplicity, reversibility, noninvasiveness, and low cost.These splints can be customized to fit either the maxillary or mandibular arch, and they can be usually used immediately. Soft splints easily disperse the severe loads experienced during parafunctional activities due to their soft and robust material properties, and they have been linked to a high level of patient tolerance. Hard splints, on the other hand, were reported to be effective in individuals with masticatory system problems. It was also saidthat both soft and hard occlusal appliances are equally effective in reducing masticatory muscle pain in the short term $[19,21]$. In light of these disagreements, the current investigation was done to compare the efficiency of splint therapy (soft versus hard occlusal splint therapies) for the management of TMDs.

According to our study, soft splint was predominantly $(89.2 \%)$ chosen for patients with TMD with only $10.8 \%$ patients were provided with hard splint as it was required.Soft splints were more effective $(70.98 \%)$ than the hard splints $(8.29 \%)$ in reducing pain and discomfort due to TMD among the patients. Splint therapy was predominantly provided for disc-condyle disorder patients followed by MPDS condition and least for the patients with degenerative disorders. In our study, splint therapy was effective in majority of the patients $(80 \%)$ with TMD. A study demonstrated thatafter splint therapy, 87 percent of patients reported less discomfort, 50\% reported lower VAS scores, and 70\% reported no clicking [22]. Soft splint therapy reduced facial myalgia and TMJ clicking by $74 \%$ in another trial [23]. The uniform intensity of interactions among all teeth, together with disocclusion of the front teeth, accounted for these advantages and both hard and soft occlusal splint therapies were effective in the treatment of TMD, while soft splint therapy improved some TMD symptoms more quickly [23].

Splint therapy for TMD symptoms was thought to be effective for at least three months. Our patients had pain relief immediately after splint therapy and it was effective for a period of 12 months. In accordance to other studies [22,23], we recommend the utility of splint therapy to treat MPDS and TMDs in individuals with anterior disc displacement and reduction.In a similar study it was found that more than half of the patients experienced total pain relief after splint therapy [24]. In another study, wearing an occlusal splint reduced TMJ pain by about $70 \%$ and muscle discomfort related with increased muscular activity by about 85.2 percent [25]. A study reported that at six weeks, 17 out of 36 patients had pain reductions of less than $50 \%$. The pain for a few participants did not subside immediately, and it took up to 6 to 12 months for them to be completely pain-free [26]. In another study, 12 patients were pain-free due to splint therapy in group 1, 5 patients were pain-free post physical therapy in group 2, and 9 patients were pain-free post physical therapy in addition to splint therapy in group 3. Splint therapy was found to be the most effective treatment approach for reducing pain and discomfort associated with TMD when compared to the other two treatments evaluated, as 12 out of 20 patients were pain-free [27].

The implantation of an occlusal splint changes the resting posture, and adjusting to this new position expands the occlusal vertical dimension beyond the open space [28]. The modified resting position improves muscle efficiency during contact and lowers muscle activity during postural duties. Meanwhile, when the vertical dimension increases, the muscular effort required reduces, resulting in reversibility. Different authors evaluated the use of a soft occlusal splint with muscle relaxants and analgesics in the treatment of MPDS, and found that occlusal splint therapy improved pain, muscle soreness, and TMJ clicking more than pharmaceutical treatment [29]. Researchers indicated that occlusal splint therapy for MPDS is effective based on data from electromyography of the masticatory muscles [29, 30]. Our findings back up their findings, demonstrating that occlusal splint therapy is a safe and effective therapeutic option for lowering pain and muscle tenderness while also enhancing jaw opening.

Previously our team had conducted numerous clinical trials [3136], and systematic reviews [37-39] regarding TMD over the past 5 years. Now we are focussing on studies to evaluate the effectiveness of various treatment modalitiesfor TMD. Limitations of the study include small sample size and a small population covered. Larger samples could be collected and analyzed over a longer time period. Future scope of this study would be to conduct a multicentric trial among diverse population. 


\section{Conclusion}

In our study, an increased number of people in the younger age, especially males received the splint therapy for the management of temporomandibular joint disorders. Soft splints were chosen for the vast majority of the people which were very effective in treating temporomandibular joint disorders. Splint therapy was very effective in treating younger age people than the elderly. Splint therapy was effective for both females and males with males exhibiting a higher response rate. Splint therapy was very effective in treating patients with disc-condyle disorder followed by MPDS patients. The association between the age and gender of the patients, types of TMD and types of splint therapy with the effectiveness of splint therapy for treating temporomandibular joint disorders were statistically significant. In our study, majority of the patients with TMJ pain responded well to splint therapy, but a small a percentage of patients developed refractory or persistent TMD.

\section{References}

[1]. van Grootel RJ, Buchner R, Wismeijer D, van der Glas HW. Towards an optimal therapy strategy for myogenous TMD, physiotherapy compared with occlusal splint therapy in an RCT with therapy-and-patient-specific treatment durations. BMC Musculoskelet Disord. 2017 Feb 10;18(1):1-7. Pubmed PMID: 28183288.

[2]. Seifeldin SA, Elhayes KA. Soft versus hard occlusal splint therapy in the management of temporomandibular disorders (TMDs). Saudi Dent J. 2015 Oct 1;27(4):208-14

[3]. Zhang L, Xu L, Wu D, Yu C, Fan S, Cai B. Effectiveness of exercise therapy versus occlusal splint therapy for the treatment of painful temporomandibular disorders: a systematic review and meta-analysis. Ann Palliat Med. 2021 May 10.Pubmed PMID: 33977737.

[4]. Okeson JP. Management of temporomandibular disorders and occlusion-Ebook. Elsevier Health Sciences; 2019 Feb 1.

[5]. Haghighat S, Oshaghi S. Effectiveness of Ozone Injection Therapy in Temporomandibular Disorders. Adv Biomed Res. 2020 Nov 28;9(1):73.Pubmed PMID: 33816392.

[6]. Boero RP. The physiology of splint therapy: a literature review. Angle Orthod. 1989 Sep;59(3):165-80

[7]. Truelove E, Huggins KH, Mancl L, Dworkin SF. The efficacy of traditional, low-cost and nonsplint therapies for temporomandibular disorder: a randomized controlled trial. J Am Dent Assoc. 2006 Aug;137(8):1099-107. Pubmed PMID: 16873325.

[8]. Yost O, Liverman CT, English R, Mackey S, Bond EC, National Academies of Sciences, Engineering, and Medicine. Improving Patient, Family, and Public Education and Awareness About TMDs. InTemporomandibular Disorders: Priorities for Research and Care 2020 Mar 12. National Academies Press (US).

[9]. Demerjian GG, Barkhordarian A, Chiappelli F, editors. Temporomandibular Joint and Airway Disorders: A Translational Perspective. Springer; 2018 Nov 3.

[10]. Manfredini D, Lobbezoo F. Relationship between bruxism and temporomandibular disorders: a systematic review of literature from 1998 to 2008. Oral Surg Oral Med Oral Pathol Oral Radiol Endod. 2010 Jun;109(6):e26-50. Pubmed PMID: 20451831

[11]. Poveda Roda R, Bagan JV, Díaz Fernández JM, Hernández Bazán S, Jiménez Soriano Y. Review of temporomandibular joint pathology. Part I: classification, epidemiology and risk factors. Med Oral Patol Oral Cir Bucal. 2007 Aug 1;12(4):E292-8.Pubmed PMID: 17664915.

[12]. Hegab AF. TMJ splint therapy; then what?. J. dent health oral disord. ther. 2015;3(2):1-2

[13]. Peixoto KO, da Silva Bezerra A, Melo RA, de Resende CMBM, de Almeida EO, Barbosa GAS. Short-Term Effect of Scalp Acupuncture on Pain, Sleep Disorders, and Quality of Life in Patients with Temporomandibular Disorders: A A Randomized Clinical Trial. Pain Med. 2021 Apr 20;22(4):905914.Pubmed PMID: 33561277.
[14]. Scrivani SJ, Keith DA, Kaban LB. Temporomandibular disorders. N Engl J Med. 2008 Dec 18;359(25):2693-705.

[15]. Palotie T, Peltomaa A, Bachour A, Bachour P, Mäkitie A, Peltomaa M, et al. Reasons for failure of mandibular advancement splint therapy in the treatment of obstructive sleep apnea. CRANIO ${ }^{\circ} .2021$ May 8:1-5.

[16]. Festa F, Rotelli C, Scarano A, Navarra R, Caulo M, Macrì M. Functional Magnetic Resonance Connectivity in Patients With Temporomadibular Joint Disorders. Front. Neurol. 2021;12.

[17]. Lee GH, Park JH, Moon DN, Lee SM. Protocols for orthodontic treatment of patients with temporomandibular joint disorders. Am J Orthod Dentofacial Orthop. 2021 Mar 1;159(3):373-88.

[18]. Lee HS, Baek HS, Song DS, Kim HC, Kim HG, Kim BJ, et al. Effect of simultaneous therapy of arthrocentesis and occlusal splints on temporomandibular disorders: anterior disc displacement without reduction. J Korean Assoc Oral Maxillofac Surg. 2013 Feb;39(1):14.

[19]. Davies SJ, Gray RJ. The pattern of splint usage in the management of two common temporomandibular disorders. Part III: Long-term follow-up in an assessment of splint therapy in the management of disc displacement with reduction and pain dysfunction syndrome. Br Dent J. 1997 Oct 25;183(8):279-83.Pubmed PMID: 9375440.

[20]. Wassell RW, Adams N, Kelly PJ. Treatment of temporomandibular disorders by stabilising splints in general dental practice: results after initial treatment. Br Dent J. 2004 Jul;197(1):35-41.

[21]. Ahmad M, Schiffman EL. Temporomandibular joint disorders and orofacial pain. Dent. Clin. N. Am. 2016 Jan;60(1):105.

[22]. Tsuga K, Akagawa Y, Sakaguchi R, Tsuru H. A short-term evaluation of the effectiveness of stabilization-type occlusal splint therapy for specific symptoms of temporomandibular joint dysfunction syndrome. J Prosthet Dent 1989 May;61(5):610-3.Pubmed PMID: 2746530.

[23]. Harkins S, Marteney JL, Cueva O, Cueva L. Application of soft occlusal splints in patients suffering from clicking temporomandibular joints. Cranio. 1988 Jan;6(1):71-6.Pubmed PMID: 3162841.

[24]. Ebrahim S, Montoya L, Busse JW, Carrasco-Labra A, Guyatt GH, Medically Unexplained Syndromes Research Group. The effectiveness of splint therapy in patients with temporomandibular disorders: a systematic review and meta-analysis. J Am Dent Assoc. 2012 Aug 1;143(8):847-57.

[25]. Carraro JJ, Caffesse RG. Effect of occlusal splints on TMJ symptomatology. The J Prosthet Dent. 1978 Nov 1;40(5):563-6.

[26]. Wassell RW, Adams N, Kelly PJ. The treatment of temporomandibular disorders with stabilizing splints in general dental practice: one-year follow-up. J Am Dent Assoc. 2006 Aug;137(8):1089-98.Pubmed PMID: 16873324.

[27]. Madani AS, Mirmortazavi A. Comparison of three treatment options for painful temporomandibular joint clicking.J. Oral Sci. 2011;53(3):349-54.

[28]. Naikmasur V, Bhargava P, Guttal K, Burde K. Soft occlusal splint therapy in the management of myofascial pain dysfunction syndrome: a follow-up study. Indian J Dent Res. 2008 Jul 1;19(3):196.

[29]. Zhang C, Wu JY, Deng DL, He BY, Tao Y, Niu YM, et al. Efficacy of splint therapy for the management of temporomandibular disorders: a meta-analysis. Oncotarget. 2016 Dec 20;7(51):84043.

[30]. Daif ET. Correlation of splint therapy outcome with the electromyography of masticatory muscles in temporomandibular disorder with myofascial pain. Acta Odontol. Scand. 2012 Jan 1;70(1):72-7.

[31]. Malay KK, Duraisamy R, Brundha MP, Kumar MP. Awareness regarding anemia among 1 st year dental undergraduate students. Drug invent. today. 2018 Aug $1 ; 10(8)$.

[32]. Kumar MS. Knowledge, attitude and practices towards oral health among law students in Chennai. j. pharm. sci. res. 2016 Jul 1;8(7):650.

[33]. Kumar MP. Dental management of patients on antiplatelet therapy: Literature update. Asian J Pharm Clin Res. 2016;9(3):26-31.

[34]. Kumar S. Newer delivery systems for local anesthesia in dentistry. J Pharm Sci Res. 2015;7(5):252-5.

[35]. Ahamed A, Kumar MS. Knowledge, attitude and perceived confidence in handling medical emergencies among dental students. j. pharm. sci. res. $2016 \mathrm{Jul} 1 ; 8(7): 645$

[36]. Kumar S. Knowledge, attitude and practices of dental students toward dental management of patients on antiplatelet therapy. Asian J Pharm Clin Res. 2016;9(30):270-6

[37]. Gayathri MM. Knowledge, Awareness and Attitude among dental students about hepatitis B infection. j. pharm. sci. res. 2016 Mar 1;8(3):168.

[38]. MP SK. Knowledge, Attitude and practices regarding needlestick injuries among dental students. Asian J Pharm Clin Res. 2016;9(4):312-5.

[39]. Sivakumar N, Sundari KK, Chandrasekar S, Kumar MP. A review on smile arc-An orthodontist's perspective. Drug invent. today. 2018 Sep 2;10. 